\title{
Qualitative and Quantitative Determination of Anti- Nutritional Factors of Five Wine Samples
}

\author{
Kingsley Ogemdi Iwuozor
}

\section{A R T I C L E I N F O}

Received: 21 January 2019

Revised: 25 January 2019

Accepted: 25 January 2019

Available online: 1 February 2019

\section{K E Y W O R D S}

Wine

Folin-Denis

Tannin

Phytate

Oxalate

\section{A B S T R A C T}

This research was aimed at the qualitative and quantitative determination of three anti-nutritional factors (Oxalate, Phytate, and Tannins) present in five wine samples produced in Nigeria. The determination of tannin was done using Folin-Denis Spectrophotometric method, the determination of oxalate was done through three processes namely; digestion, oxalate precipitation and permanganate titration. The result revealed that the five wine samples actually contained these anti-nutritional factors and these compounds were present in amounts that were not toxic to the human health. Ingestion of the wine samples over a long period of time could increase the anti-nutritional content and cause serious health challenges that could affect the liver and kidney. Therefore sensitization on the harms of prolonged intake of these wine samples should be a priority to the government. Government should also impose a strict law that will checkmate manufacturers of these drinks so as to make sure that these drinks are properly treated.

\section{GRA P H ICAL ABSTRACT}
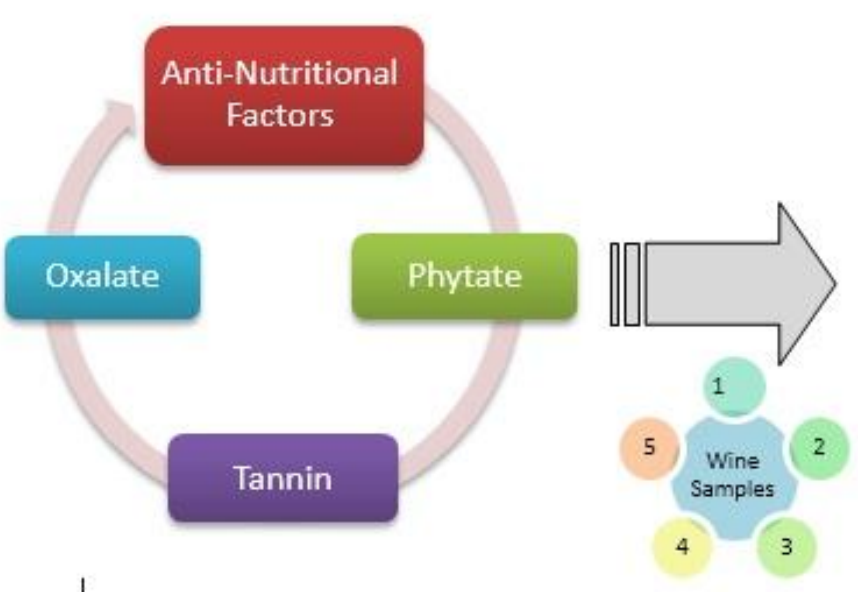

\begin{tabular}{|c|c|c|}
\hline $\begin{array}{c}\text { Wine } \\
\text { Sample }\end{array}$ & $\begin{array}{c}\text { Absorbance } \\
(\mathbf{n m})\end{array}$ & $\begin{array}{c}\text { Tannin } \\
(\%)\end{array}$ \\
\hline Sample 1 & 0.258 & 6.5 \\
\hline Sample 2 & 0.005 & 0.1 \\
\hline Sample 3 & 0.248 & 6.3 \\
\hline Sample 4 & 0.231 & 5.9 \\
\hline Sample 5 & 0.111 & 2.8 \\
\hline
\end{tabular}

\footnotetext{
* Corresponding author's E-mail address: kingsleyiwuozor5@gmail.com, Tel.: +2347034810425

Department of pure and industrial chemistry, Nnamdi Azikiwe University, Awka, Nigeria.
} 


\section{Introduction}

Nutrients are the chemical substances supplied by food that the body needs for growth, maintenance and repair [1]. They include; Carbohydrates, protein, minerals, fats and oils, vitamins and water. Nutrients are considered either essential or nonessential depending on whether the body can or cannot manufacture them. When the body requires a nutrient for growth or maintenance but lack the ability to manufacture it in amounts sufficient to meet bodily needs, this essential nutrient must be supplied by foods in the diet [1]. All nutrients perform one or more of the following functions; Serve as source of energy or heat, Support the growth and maintenance of body tissues, Aid in regulation of basic body processes. These three life sustaining functions collectively are part of metabolism, the sum of all physical and chemical changes that take place in the body. Nutrients have specific metabolic functions and interact with one another to maintain the body. Ingesting too much or too little of nutrient can interfere with health and well-being. There is a beneficial range of intake of any nutrient, an intake below or above that range which is incompatible with optimal health.

Anti-nutritional factors are those substances or chemical compounds found in fruits and food substances in general which are poisonous to humans or in some ways limit the nutrient availability to the body. Antinutritional factor are present in different food substances in varying amounts depending on the kind of food, mode of its propagation, chemicals used in growing the crops as well as those chemicals used in storage and preservation of the food substances. These anti-nutritional factors are known to interfere with metabolic processes such that growth and bioavailability of nutrients are negatively influenced $[2,3]$.

The anti-nutritional factors may be defined as those substances generated in natural food stuffs by the normal metabolism of species and by different mechanisms (e.g. inactivation of some nutrients, diminution of the digestive process, or metabolic utilization of feed) which exert effects contrary to optimum nutrition [3].

The knowledge that these compounds elicit both toxic and advantageous biological responses has given rise to several investigations in recent times as to their possible physiological implications in various biological systems. It is well known that plants generally contain anti-nutrients acquired from fertilizer and pesticides and several naturally-occurring chemicals. Some of these chemicals are known as "secondary metabolites" and they have been shown to 
be highly biologically active. They include saponins, tannins, flavonoids, alkaloids, trypsin (protease) inhibitors, oxalates, phytates, haemaggluttinins (lectins), cyanogenic glycosides, cardiac glycosides, coumarins and gossypol. The list is inexhaustible.

There is a wide distribution of biologicallyactive constituents throughout the plant kingdom, particularly in plants used in human nutrition [2]. The knowledge that these compounds elicit both toxic and advantageous biological responses have given rise to several investigations in recent times as to their possible physiological implications in various biological systems [2]. It is well known that plants generally contain anti-nutrients acquired from fertilizers and pesticides and several naturally-occurring chemicals [2]. Some of these chemicals are known as "secondary metabolites" and they have been shown to be highly biologically active [4]. They include Saponins, Tannins, Flavonoids, Alkaloids, Trypsin (Protease) Inhibitors, Oxalates, Phytates, Haemaggluttinins (Lectins), Cyanogenic Glycosides, Cardiac Glycosides, Coumarins and Gossypol. The list is inexhaustible.

Some of these plant chemicals have been shown to be deleterious to health or evidently advantageous to human and animal health if consumed at appropriate amounts [5, 6]. Most of these secondary metabolites elicit very harmful biological responses, while some are widely applied in nutrition and as pharmacologically-active agents $[7,8]$. The pharmacological and other beneficial effects of these anti-nutritional factors in plants have been reviewed by [8]. Much of the available data and information on the nutrient and anti-nutrient composition of the more commonly used local foods do not cover all the foods available, so they need updating. This is because of the possible effects of variety/genetic origin, climate, soil, processing methods, pesticides and fertilizers on the chemical composition of the food plants [9]. Several considerations justify the continued surveillance, knowledge and future research on antinutritional factors/toxic substances naturally present in plants used as foods and ways of reducing them to safe level of consumption.

The possibility that newly developed or exotic foods/drinks may contain natural toxicants must be taken into account in evaluating their usefulness [10]. Introduction of new plant varieties into our diets may expose humans and animals to new toxic factors with unsuspected biological effects. Also, information on the 
anti-nutritional components of foods will help dieticians, veterinarians, human and animal nutritionists to avoid recommending foods that their patients cannot tolerate, possibly because of the inability to metabolize or detoxify certain substances therein.

The anti-nutritional factors may be classified on the basis of their chemical structure, the specific actions they bring about or their biosynthetic origin. Although this classification does not encompass all the known groups of anti-nutritional factors, it does present the list of those frequently found in human foods and drinks. The antinutritional factors may be divided into two major categories, they are; Proteins (such as lectins and protease inhibitors) which are sensitive to normal processing temperatures. Other substances which are stable or resistant to these temperatures and which include, among many others, polyphenolic compounds (mainly condensed tannins), non-protein amino acids and galactomannan gums [10].

More often than not, a single plant may contain two or more toxic compounds, generally drawn from the two categories, which add to the difficulties of detoxification. According to [11], there are several anti-nutritional factors that are very significant in plants used for human foods, drinks and animal feeds. They are: (I)
Enzyme inhibitors (trypsin and chymotrypsin inhibitors, plasmin inhibitors, elastase inhibitors), (ii) Haemagglutinnins (concanavalin A, ricin), (iii) Plant enzymes (urease, lipoxygenase), (iv) Cyanogenic glycosides (phaseolunatin, dhurrin, linamarin, lutaustralin), (v) Goitrogens (progoitrins and glucosinolates), (vi) Oestrogens (flavones and genistein), (vii) Saponins (soya sapogenin), (viii) Gossypol from Gossypium species e.g. cotton, (ix) Tannins (condensed and hydrolysable tannins), (x) Amino acid analogues (BOAA, DAP, mimosine, $\quad N$-methyl-1-alanine), (xi) Alkaloids (solanine and chaconine), (xii) Anti-metals (phytates and oxalates), (xiii) Anti-vitamins (anti-vitamins A, D, E and B12) and (xiv) Favism factors.

Polyphenols: are groups of chemical substances present in plants (fruits, vegetables). Phenolic compounds are responsible for the color of many plants, such as apples, they are part of the taste and flavor of beverages (apple juice, tea), and are important anti-oxidants in plants. Polyphenols are normally complex organic substances, which contain more than one phenol group. Polyphenols can be divided into many different sub categories, such as anthocyans (colors in fruits), flavonoids (catechins, tannins in tea and wine) and nonflavonoids components (gallic acid in tea leaves). Flavonoids are formed in plants 
from the aromatic amino acids phenylalanine and tyrosine [12].

Cyanogens: are glycosides of a sugar, or sugars, and cyanide containing a glycone. According to [13], Cyanogens can be hydrolyzed by enzymes to release HCN which is a volatile gas. However, the glycosides occur in vacuoles in plant cell and enzymes are found in the cytosol. Damage to the plants results in the enzymes and glycoside coming together and producing HCN. The hydrolytic reaction can take place in the rumen by microbial activity. Hence ruminants are more susceptible to $\mathrm{HCN}$ toxicity than non-ruminants. The HCN is absorbed and is rapidly detoxified in the liver by the enzyme Rhodanese which converts HCN to Thiocyanate (SCN). Excess cyanide ion inhibits the cytochrome oxidase. This stops ATP formation, tissues suffer energy deprivation and death follows rapidly. Recorded accounts of livestock poisoning by cyanogenic plants show that such situations do arise. Cyanide can cause goitrogenic effects due to thiocyanate produced during detoxification. Poor animal performance due to A. sieberiana pod feeding has been attributed to cyanogens. Animals suffering from cyanide must be immediately treated by injecting suitable dose of sodium nitrate and sodium thiosulphate.
Saponins: are glycosides containing a polycyclic a-glycone moiety of either $C_{27}$ steroid or C", triterpenoid (collectively termed as sapogenins) attached to a carbohydrate [14]. Saponins are characterized by a bitter taste and foaming properties. Erythrocytes lyse in saponin solution and so these compounds are toxic when injected intravenously. The antinutritional effects of saponins have been mainly studied using alfalfa saponins [14]. In non-ruminants (chicks and pigs), retardation of growth rate, due primarily to reduction in feed intake, is probably the major concern. Such effects have also been noted when Sesbania sesban leaf meal (saponin $0.71 \%$ ) was incorporated in a chick diet. In ruminants, saponins were implicated in causing bloat. However, later studies indicate that they are not involved in the bloat syndrome. Furthermore, because saponins may also undergo bacterial degradation in the rumen, they may not retard the growth of ruminants. Nevertheless, recent studies indicate that they inhibit microbial fermentation and synthesis in the rumen. In ruminants, some reports of toxicity due to dietary saponins have also appeared. It was observed that 47 weeks feeding of Albizia stipulata gave rise to toxic manifestations in sheep. The toxicity of broom breed (Gulierrezia sarolhrae), a resinous shrub, is believed to be due to its 
saponin content. Symptoms include listlessness, anorexia, weight loss and gastroenteritis. These results indicate that saponins from different plant species have varied biological effects probably due to structural differences in their sapogenin fractions. The adverse effects of saponins can be overcome by repeated washing with water which makes the feed more palatable by reducing the bitterness associated with saponins.

Phytohemagglutinins: otherwise referred to as lectins, according to [15] are proteins which agglutinate red blood cells. They have been shown to occur in some important fodder trees. The highest concentrations of lectins are found in seeds but, in the leaves, their concentration is low due to translocation. The biological effects of lectins probably result from their affinity for sugars. They may bind to the carbohydrate moieties of cells of the intestinal wall and cause a non-specific interference with nutrient absorption. In fodder trees, the lectins of interest are robin and ricin. Robin, a lectin from Robinia pseudoacacia, has been reported to cause symptoms of anorexia, lassitude, weakness and posterior paralysis [15]. Ricin occurs in castor beans (Ricinus communis) which have been reported to cause poisoning in all class of livestock.

Tannins: are water soluble phenolic compounds with a molecular weight greater than 500 and with the ability to precipitate proteins from aqueous solution [16]. They occur almost in all vascular plants. Hydrolysable tannins and condensed tannins (proanthocyanidins) are two different groups of these compounds. Generally, tree and shrub leaves contain both types of tannins. The two types differ in their nutritional and toxic effects. The condensed tannins have a more profound digestibility-reducing effect than hydrolysable tannins, whereas the latter may cause varied toxic manifestations due to hydrolysis in rumen. Tannins form insoluble complexes with proteins, carbohydrates and lipids leading to a reduction in digestibility of these nutrients. Other nutritional effects that have been attributed to tannins include damage to the intestinal tract, toxicity of tannins absorbed from the gut, interference with the absorption of iron and a possible carcinogenic effect.

Phytic acid: lowers the utilization of elements like calcium, magnesium, zinc and iron due to its ability to form insoluble salts with their ions. The phytic acid content of 64 foodstuffs was analyzed and the result showed that $20-60 \%$ of the phytates in cereals excreted by human beings are unchanged in the faeces.

Oxalate: is produced and accumulated in many crop plants and pasture weeds. When 
taken into the body, oxalates do not only combine with calcium but also with magnesium to form insoluble salts which are not available to the body.

The aim of this study was to evaluate the anti-nutritional factors present in five different brands of wines produced in Nigeria while the objectives are as follows; to obtain quantitative data on the antinutritional factors present in the wine samples obtained; to comparatively analyze the anti-nutritional factors found in the wine samples; to emphasize the need to properly process plant products before consumption so as to rid them of such anti-nutrients.

\section{Materials and methods}

All the reagents used for this research were of analytical grade. The reagents as well as the samples were purchased from Bridge Head, Onitsha. The expiry dates confirmed that the wines were not yet expired. The UV spectrophotometer was greenway model 6000.

\section{Methods}

\section{Determination of tannin}

\section{Folin-Denis spectrophotometric method}

A measured weight of each sample $(1.0 \mathrm{~g})$ was dispersed in $10 \mathrm{~mL}$ distilled water and agitated. This was allowed to stand for $30 \mathrm{mins}$ at room temperature while continuously stirring every 5 mins. At the end of 30 mins, it was centrifuged and the extract obtained. $2.5 \mathrm{~mL}$ of the extract was dispersed into a $50 \mathrm{~mL}$ volumetric flask. Similarly, $2.5 \mathrm{~mL}$ of standard tannic acid was dispersed into a separate $50 \mathrm{ml}$ flask. A $1.0 \mathrm{~mL}$ Folin-Denis reagent was measured into each flask followed by the addition of $2.5 \mathrm{~mL}$ of saturated $\mathrm{Na}_{2} \mathrm{CO}_{3}$ solution. The mixture was diluted and made up to the $50 \mathrm{~mL}$ mark of the flask and was incubated for 90 mins at room temperature. The absorbance was measured at $250 \mathrm{~nm}$ in a UV spectrophotometer; readings were taken with the blank sample at zero.

The tannin content was given as follows;

$\%$ Tannin $=$ An/As $\times \mathrm{C} \times 100 / \mathrm{w} \mathrm{x} \mathrm{V}_{\mathrm{F}} / \mathrm{V}_{\mathrm{A}}$

$\mathrm{An}=$ absorbance of the test sample

As = absorbance of standard solution

$\mathrm{C}=$ concentration of standard solution

$\mathrm{W}=$ weight of sample used

$V_{F}=$ total volume of extract

$\mathrm{V}_{\mathrm{A}}=$ volume of extract analyzed

\section{Oxalate Determination}

This determination involved three major steps: digestion, oxalate precipitation, and permanganate titration.

\section{Digestion}

$2 \mathrm{~g}$ of the sample was suspended in $190 \mathrm{~mL}$ of distilled water in a $250 \mathrm{~mL}$ volumetric flask. 10 $\mathrm{mL}$ of $6 \mathrm{M} \mathrm{HCl}$ was added and the suspension digested at $100{ }^{\circ} \mathrm{C}$ for 1 hour. The solution was cooled, and then made up to $250 \mathrm{~mL}$ mark before filtration. 


\section{Oxalate Precipitation}

Duplicate Portions of the filtrate were measured into breakers and four drops of methyl red indicator added. Then $\mathrm{NH}_{4} \mathrm{OH}$ solution was added (drop wise) until the test solution changed from pink to faint yellow colour $(\mathrm{pH}$ 4.0-4.5). Each portion was then heated to $90{ }^{\circ} \mathrm{C}$, cooled and then filtered to remove the precipitate containing ferrous ion. The filtrate was again heated to $90^{\circ} \mathrm{C}$ and $10 \mathrm{ml}$ of $5 \% \mathrm{CaCl}_{2}$ solution was added while being stirred constantly. The solution was then heated and left overnight at $25^{\circ} \mathrm{C}$, it was then centrifuged at 2000rpm for 5minutes. The supernatant was decanted and the precipitate completely dissolved in $20 \mathrm{ml}$ of $25 \%$ (v/v) $\mathrm{H}_{2} \mathrm{SO}_{4}$ solution.

\section{Permanganate Titration}

At this point, the total filtrate resulting from the digestion of $2 \mathrm{~g}$ of sample was made up to a volume of $300 \mathrm{~mL}$. Aliquots of $125 \mathrm{~mL}$ of the filtrate was heated until near boiling and then titrated against $0.02 \mathrm{M}$ standardized $\mathrm{KMnO}_{4}$ solution to a faint pink color which persisted for 30 seconds. The calcium oxalate content was calculated using the formula;

$$
\frac{T \times\left(V_{m e}\right) \times\left(D_{f}\right)}{M_{e} \times M_{f}}(M g / 100 g)
$$

Where $\mathrm{T}$ is the titre of $\mathrm{KMnO}_{4}(\mathrm{~mL})$,

$\mathrm{V}_{\mathrm{me}}$ is the volume-mass equivalent

$D_{\mathrm{f}}$ is the Dilution factor $=\mathrm{Vt} / \mathrm{A}$ Where $\mathrm{Vt}$ is the total volume of filtrate $(300 \mathrm{~mL})$ and $A$ is the aliquot used i.e. $125 \mathrm{~mL}$,

$\mathrm{M}_{\mathrm{e}}$ is the molar equivalent of $\mathrm{KMnO}_{4}$ in oxalate and

$\mathrm{M}_{\mathrm{f}}$ is the mass of sample used.

\section{Phytate Determination}

$5 \mathrm{~mL}$ of the sample was mixed, cured for 5 hrs and filtered. Aliquots of $2500 \mathrm{~mL}$ of the filtrate in a conical flask was added to 5.00 $\mathrm{mL}$ of $0.30 \%$ ammonium thiocynate, the mixture was titrated with standard iron (III) chloride solution to a persistent brownishyellow coloration that persisted for 4 mins.

The amount of phytates was calculated with the equation below;

$$
\text { Phytic acid }=\frac{\text { Titre value } \times 0.00195 \times 1.9 \times 100}{2}
$$

Table 1. Table showing the Oxalate Content of the five wine samples

\begin{tabular}{ccccc}
\hline Wine Samples & Initial reading & Final reading & Average titre & Oxalate content \\
\hline Sample 1 & 1.0 & 13.2 & 12.2 & 0.082 \\
Sample 2 & 2.3 & 7.4 & 5.1 & 3.366 \\
Sample 3 & 2.0 & 27.2 & 7.2 & 4.752 \\
Sample 4 & 27.2 & 37.3 & 10.1 & 6.666 \\
Sample 5 & 5.0 & 15.2 & 10.2 & 6.732 \\
\hline
\end{tabular}


Table 2. Table showing the Phytic Acid content of the five wine samples

\begin{tabular}{ccccc}
\hline Wine Samples & Initial reading & Final reading & Average titre & Phytic acid content \\
\hline Sample 1 & 0.3 & 0.02 & 0.16 & 0.02964 \\
Sample 2 & 0.2 & 0.1 & 0.15 & 0.02778 \\
Sample 3 & 0.05 & 0.05 & 0.05 & 0.00926 \\
Sample 4 & 0.05 & 0.05 & 0.05 & 0.00926 \\
Sample 5 & 0.05 & 0.05 & 0.05 & 0.00926 \\
\hline
\end{tabular}

Table 3. Determination of Tannins

\begin{tabular}{ccc}
\hline Wine Sample & Absorbance (nm) & Tannin content (\%) \\
\hline Sample 1 & 0.258 & 6.5 \\
Sample 2 & 0.005 & 0.1 \\
Sample 3 & 0.248 & 6.3 \\
Sample 4 & 0.231 & 5.9 \\
Sample 5 & 0.111 & 2.8 \\
\hline
\end{tabular}

\section{Results and discussion}

From Table 1 above, it could be observed that Sample 5 wine sample had the highest content of o-xalate (6.732), while sample 1 had the lowest (0.082). Oxalic acid is an organic acid produced in animals and plants when sugar, carbohydrates and other carbon sources are metabolized. Oxalic acid does not circulate freely in the body; it links to sodium or potassium in a soluble oxalatesalt form. But when oxalic acid finds itself in the presence of calcium, it has the ability to link to it too, forming a particular insoluble salt crystal known as calcium oxalate.

A distinctive property of oxalic acid that makes it so dangerous is that once it has linked with calcium, it is practically insoluble at the acidic $\mathrm{pH}$ normally found within the body. An unusual characteristic of calcium oxalate is that nothing can dissolve it and that makes it such an exasperating problem. Over consumption of food or drinks with high concentration of oxalate causes kidney stones. The level of oxalate in the samples analyzed is not high to pose any health treat.

From Table 2 above, it could be seen that Sample 1 has the highest content of phytic acid (0.02964), followed closely by Sample 2 (0.02778), while Sample 3, Sample 4 and Sample 5 fruit wines have the lowest phytic acid contents (0.00926) each.

Phytate is the salt form of phytic acid known as inositol hexakisphosphate (IP6). Phytin refers specifically to the calcium or magnesium salt form of phytic acid. It is the principal storage form of phosphorus in many plant tissues, especially bran and seeds. Phytate is not digestible by humans or non-ruminant animals, so it is not a source 
of either inositol or phosphate if eaten directly. It has the ability to chelate that is to form complexes with proteins and inhibits the enzymatic digestion of injected protein. Phytic acid chelates, makes unobservable certain important micronutrients such as zinc and iron, and to a lesser extent, also macronutrients such as calcium and magnesium. Phytic acid also has some antinutritional properties due to its ability to lower the bioavailability of some essential minerals such as zinc and iron, and to a lesser extent, the calcium and the magnesium in the digestive tract resulting in mineral deficiencies. The phytate composition of the samples analyzed is low and might not pose any health hazard.

From Table 3 above, it was found that Sample 1 had the highest tannin content of $6.3 \%$ while Sample 2 had the lowest tannin content of $0.1 \%$. Sample 3 followed Sample 1 with tannin content of $6.3 \%$, Sample 4 had a content of $5.9 \%$ and Sample 5 had a content of $2.8 \%$.

Tannins are aromatic compounds containing phenolic groups. They interact with salivary proteins and glycoproteins in the mouth and render the tissues astringent to taste. Astringency gives tannin the medicinal value in preventing diarrhea and dysentery and for controlling hemorrhage. They also protect plants against dehydration and rotting. When tannins polymerize, they form a protective barrier against microbial attack.

\section{Conclusion}

Based on the results of this research and the subsequent discussion, it has been revealed that the Wine samples actually contain antinutritional factors but the amounts present are below toxic levels. Hence, these wine samples are good for human consumption and its anti-nutrients levels do not have any adverse effect on the human health. Though in small amounts, these anti- nutritional factors in the selected wine samples if taken for a long period may cause some serious health issues such as kidney disease and other ailments; therefore, I recommend that manufacturers of these drinks realize that these drinks are properly treated to eliminate entirely these anti- nutritional factors and that government should impose a strict law that will checkmate manufacturers of these drinks so as to make sure that these drinks are properly treated.

\section{ORCID}

Kingsley Ogemdi Iwuozor (D): 0000-0002-1161$\underline{2147}$

\section{References}

[1]. S.R. Mudambi, Fundamentals of foods and nutrition. New Age International. 2001, pp. $45-175$.

[2]. G.O. Igile, Ph.D thesis, Department of Biochemistry, University of Ibadan, Nigeria, 
1996, pp. 55-60.

[3]. R. Kumar, Proceedings of the FAO Expert

Consultation held at the Malaysian

Agricultural Research and Development

Institute (MARDI) in Kuala Lumpur,

Malaysia, 1992, pp. 14-18.

[4]. M.H. Zenk, Phytochemistry, 1991, 30,

3861-3863.

[5]. G.F. Kersten, A. Spiekstra, E.C. Beuvery,

D.J. Crommelin, Biochim. Biophys. Acta,

Biomembr.1991, 1062, 165-171.

[6]. M. Sugano, S. Goto, Y. Yamada, K.

Yaoshida, Y. Hashimoto, T. Matsno, and M.

Kimoto, J. Nutrit., 1993, 120, 977-985.

[7]. D. Oakenfull, G.S. Sidhu, P.R Cheek,

Saponins: In Toxicants of plant origin, CRC.

Press Inc. Florida, 1989, p.97.

[8].K.O. Soetan, African J. Biotechnol., 2008,

7, 4713-4721.

[9]. FAO. Food and Agricultural
Organization, conservation guide 19, Rome, 1988, pp. 333-335.

[10]. A.U. Osagie. Antinutritional Factors. In: Nutritional Quality of Plant Foods, Ambik Press Ltd, Benin City, Nigeria, 1998, pp. 1$40 ; 221-244$.

[11]. V.A. Alertor, Vet. Hum. Toxicol., 1993, $35,57-67$.

[12]. T.C. Jones, R.C. Hunt, N.W. King, Veterinary Pathology, 1997, p. 433.

[13]. S.A. Adeniyi, C.L. Orjiekwe, J.E. Ehiagbonare, African J. Biotechnol., 2009, 8, 110-112.

[14]. Y. Fujita, I. Mori, K. Fujita, T. Tanaka, Y. Koshiyama, H. Kawabe, Chem. Pharmac. Bull., 1986, 34, 2236-2238.

[15]. B.L. Salako, African J. Med. Med. Sci., 2005, 34, 335-340.

[16]. P.N. Okafor, E. Nwogbo, African J. Biotechnol., 2005, 4, 1105-1108.

How to cite this manuscript: Kingsley Ogemdi Iwuozor, Qualitative and Quantitative Determination of Anti-Nutritional Factors of Five Wine Samples, Adv. J. Chem. A, 2019, 2(2), 136-146. 